\title{
Polymorphisms of TNF- $\alpha$-308 G/A and IL-8 -251 T/A Genes Associated with Urothelial Carcinoma: A Case-Control Study
}

\author{
Chia-Chang Wu, ${ }^{1,2,3}$ Yung-Kai Huang $\mathbb{D}^{4},{ }^{4}$ Chao-Yuan Huang, ${ }^{5}$ Horng-Sheng Shiue, \\ Yeong-Shiau Pu, ${ }^{5}$ Chien-Tien Su $\oplus^{1,7}$ Ying-Chin Lin, ${ }^{8,9,10}$ and Yu-Mei Hsueh $\oplus^{10,11}$ \\ ${ }^{1}$ School of Public Health, College of Public Health and Nutrition, Taipei Medical University, Taipei 110, Taiwan \\ ${ }^{2}$ Department of Urology, School of Medicine, College of Medicine, Taipei Medical University, Taipei 110, Taiwan \\ ${ }^{3}$ Department of Urology, Shuang Ho Hospital, Taipei Medical University, New Taipei City 235, Taiwan \\ ${ }^{4}$ School of Oral Hygiene, College of Oral Medicine, Taipei Medical University, Taipei 110, Taiwan \\ ${ }^{5}$ Department of Urology, National Taiwan University Hospital, College of Medicine, National Taiwan University, Taipei 100, Taiwan \\ ${ }^{6}$ Department of Chinese Medicine, Chang Gung Memorial Hospital and Chang Gung University, College of Medicine, \\ Taoyuan 333, Taiwan \\ ${ }^{7}$ Department of Family Medicine, Taipei Medical University Hospital, Taipei Medical University, Taipei 110, Taiwan \\ ${ }^{8}$ Department of Family Medicine, School of Medicine, College of Medicine, Taipei Medical University, Taipei 110, Taiwan \\ ${ }^{9}$ Department of Health Examination, Wan Fang Hospital, Taipei Medical University, Taipei 116, Taiwan \\ ${ }^{10}$ Department of Family Medicine, Shuang Ho Hospital, Taipei Medical University, New Taipei City 235, Taiwan \\ ${ }^{11}$ Department of Public Health, School of Medicine, College of Medicine, Taipei Medical University, Taipei 110, Taiwan
}

Correspondence should be addressed to Yu-Mei Hsueh; ymhsueh@tmu.edu.tw

Received 5 February 2018; Revised 1 April 2018; Accepted 10 April 2018; Published 21 May 2018

Academic Editor: Sajib Chakraborty

Copyright (C) 2018 Chia-Chang Wu et al. This is an open access article distributed under the Creative Commons Attribution License, which permits unrestricted use, distribution, and reproduction in any medium, provided the original work is properly cited.

\begin{abstract}
Cigarette smoking and exposure to environmental tobacco smoke are well-known risk factors for urothelial carcinoma (UC). We conducted a hospital-based case-control study involving $287 \mathrm{UC}$ cases and 574 cancer-free controls to investigate the joint effects of cigarette smoking and polymorphisms of inflammatory genes on UC risk. Tumor necrosis factor alpha (TNF- $\alpha$ ) -308 G/A and interleukin-8 (IL-8) -251 T/A polymorphisms were determined using a polymerase chain reaction-restriction fragment length polymorphism method. People who had ever smoked and those who were exposed to environmental tobacco smoke had significantly increased UC odds ratios (ORs) of 1.65 and 1.68, respectively. Participants who had smoked more than 18 pack-years had a significantly increased UC OR of 2.64. People who had ever smoked and who carried the A/A genotype of the TNF- $\alpha-308$ G/A polymorphism had a significantly higher UC OR (10.25) compared to people who had never smoked and who carried the G/G or G/A genotype. In addition, people who had ever smoked and who carried the $I L-8-251 \mathrm{~T} / \mathrm{T}$ genotype had a significantly increased UC OR (3.08) compared to people who had never smoked and who carried the T/A or A/A genotype. In a combined analysis of three major risk factors (cumulative cigarette smoking, the TNF- $\alpha-308 \mathrm{~A} / \mathrm{A}$ genotype, and the $I L-8-251 \mathrm{~T} / \mathrm{T}$ genotype), subjects with any one, any two, and all three risk factors experienced significantly increased UC ORs of 1.55, 2.89, and 3.77, respectively, compared to individuals with none of the risk factors. Conclusions. Our results indicate that the combined effects of cumulative cigarette exposure and the TNF- $\alpha-308 \mathrm{~A} / \mathrm{A}$ genotype and/or the IL-8 -251 T/T genotype on UC OR showed a significant doseresponse relationship.
\end{abstract}

\section{Introduction}

The bladder is the most common site of urothelial carcinoma (UC), a multifactorial malignancy influenced by exogenous exposure to environmental risk factors and molecular variations in metabolism-related genes [1]. Cigarette smoking is the major risk factor for bladder cancer, and epidemiological studies have indicated that cigarette smokers have a 2 4fold increased risk of bladder cancer [2]. Cigarettes contain approximately 60 chemical carcinogens, including polycyclic 
aromatic hydrocarbons, aromatic amines, and N-nitroso compounds, which are associated with the development of bladder cancer [3]. Cigarette smoke contains free radicals and induces oxidative stress, which is associated with the development of bladder cancer $[4,5]$.

Oxidative stress can induce proinflammatory cytokines such as tumor necrosis factor alpha (TNF- $\alpha$ ) and interleukin8 (IL-8), which are involved in the development of various malignancies [6,7]. Exposure to cigarette smoke can enhance TNF- $\alpha$ expression through upregulating the activator protein, AP-1 [8]. TNF- $\alpha$ is involved in the immune response during the intravesical instillation of bacillus CalmetteGuerin [9]. Previous studies reported that cigarette smoking can induce inflammation that is characterized by increased levels of cytokines such as TNF- $\alpha$ and IL- $8[10,11]$. IL- 8 is a proinflammatory chemokine that is secreted by various cell types. Studies showed the chronic inflammation is involved in various tumorigenesis steps. TNF- $\alpha$ initiates signaling pathways that activate proinflammatory gene expression via the transcription factor; nuclear factor-kappa B (NF- $\kappa \mathrm{B})$ is also produced by tumors and acts as an endogenous tumor promoter. Cytokines, like IL-6 and IL-8, are also involved in transformation and angiogenesis, respectively [12]. Environmental-genetic associations with tobacco smoke combined with genetic polymorphisms of inflammationrelated genes provide additional bladder cancer risk.

The polymorphism of TNF- $\alpha$ gene (-308 G/A, rs1800629) is located in promoter and has functional impact on the TNF$\alpha$ expression [13-15]. Compared to SNPs of TNF- $\alpha$, -308 G/A polymorphism was investigated and found to be related to several cancers, including breast, gastric, and bladder cancers [13, 16-18]. However, results from previous studies were inconsistent. The $I L-8$ gene is located on chromosome $4 \mathrm{q} 13$ $\mathrm{q} 21$, and several polymorphisms were identified in this gene [19]. A common polymorphism at the promoter region (-251 $\mathrm{T} / \mathrm{A}$, rs4073) was reported to influence the transcriptional activity of $I L-8$, and it was identified to be associated with various cancer risks in Asian group [6]. Therefore, these findings raise the possibility that genetic variations in the $T N F-\alpha$ and $I L-8$ genes may modify the risk of UC.

To investigate the effects of TNF- $\alpha-308 \mathrm{G} / \mathrm{A}$ and $I L-8-251$ T/A polymorphisms on the UC risk, we conducted a hospitalbased case-control study. We also examined the combined effects of cumulative cigarette exposure, the TNF- $\alpha-308 \mathrm{~A} / \mathrm{A}$ genotype, and the $I L-8-251 \mathrm{~T} / \mathrm{T}$ genotype on UC risk.

\section{Material and Methods}

2.1. Study Population. This study involved 861 participants enrolled from the Departments of Urology and those undergoing a general health examination at National Taiwan University Hospital, Taipei Medical University Hospital, and Taipei Municipal Wan Fang Hospital between September 2002 and May 2009. In total, 287 UC cases were included (with a mean age of $62.95 \pm 13.62$ years). Each case was diagnosed with histopathological confirmation, which was performed using routine urological practices and verified by board-certified pathologists. In total, 574 age- and gendermatched cancer-free controls (with a mean age of $62.56 \pm$
13.50 years) were recruited from a hospital-based pool. All participants provided informed consent before the questionnaire interview and biospecimen collection. The Research Ethics Committee of National Taiwan University Hospital (Taipei, Taiwan) approved this study, and this study complied with the World Medical Association Declaration of Helsinki.

\subsection{Questionnaire Interview and Biospecimen Collection.} Well-trained investigators interviewed each patient using a structured questionnaire. Information collected by the questionnaire included demographic characteristics and lifestyle habits such as cigarette smoking, secondhand smoke exposure, betel nut chewing, alcohol, tea, and coffee consumption, exposure to occupational and environmental carcinogens such as pesticides, and a family history of disease. Study subjects who had smoked more than 100 cigarettes during their lifetime were regarded as people who had ever smoked, while those who had smoked fewer than 100 cigarettes were defined as people who had never smoked. Environmental tobacco smoke (secondhand smoke exposure) was assessed through a questionnaire asking participants whether anyone had ever smoked around them. A 6 8-ml sample of venous blood was drawn from each participant for genotype determination.

2.3. Genotyping of the TNF- $\alpha-308$ G/A and IL-8 -251 T/A Polymorphisms. Genomic DNA was extracted from the buffy coat of peripheral blood using standard methods and stored at $-80^{\circ} \mathrm{C}$. Genotyping was completed using a polymerase chain reaction-restriction fragment length polymorphism (PCR-RFLP) method modified from a study by Duarte et al. [20]. Briefly, the following primer sets were designed for the TNF- $\alpha-308$ G/A polymorphism: $5^{\prime}$-TCCTCCCTGCTCCGATTCCG-3' (sense) and $5^{\prime}$-AGG CAATAGGTTTTGAGGGCCAT- $3^{\prime}$ (antisense). The thermal PCR conditions were as follows: one cycle at $95^{\circ} \mathrm{C}$ for $5 \mathrm{~min}$; 35 cycles of $95^{\circ} \mathrm{C}$ for $30 \mathrm{~s}, 58^{\circ} \mathrm{C}$ for $30 \mathrm{~s}$, and $72^{\circ} \mathrm{C}$ for $45 \mathrm{~s}$; and a final extension at $72^{\circ} \mathrm{C}$ for $10 \mathrm{~min}$. After complete digestion with the NcoI restriction enzyme, the resulting DNA fragments, which represented the TNF- $\alpha-308$ G/A polymorphism, were determined (G/G: 87 and 20 bp; G/A: 107, 87, and $20 \mathrm{bp}$; and A/A: $107 \mathrm{bp}$ ). PCR-RFLP genotyping and sequencing of TNF- $\alpha-308$ G/A are shown in Figure 1(a). For the $I L-8-251 \mathrm{~T} / \mathrm{A}$ polymorphism, the following primers were used: $5^{\prime}$-ATCTTGTTCTAACACCTGCCACTC-3' (sense) and $5^{\prime}$-TAAAATACTGAAGCTCCACAATTTGG-3' (antisense) [21]. The PCR conditions were as follows: one cycle at $94^{\circ} \mathrm{C}$ for $5 \mathrm{~min} ; 35$ cycles of $94^{\circ} \mathrm{C}$ for $50 \mathrm{~s}, 61^{\circ} \mathrm{C}$ for $60 \mathrm{~s}$, and $72^{\circ} \mathrm{C}$ for $55 \mathrm{~s}$; and a final extension at $72^{\circ} \mathrm{C}$ for $5 \mathrm{~min}$. The genotypes were determined after digestion with the MfeI restriction enzyme for the $I L-8-251 \mathrm{~T} / \mathrm{A}$ polymorphism $(\mathrm{T} / \mathrm{T}$ : $121 \mathrm{bp}$; T/A: 121, 82, and $39 \mathrm{bp}$; and A/A: 82 and $39 \mathrm{bp)}$. PCRRFLP genotyping and sequencing of $I L-8-251 \mathrm{~T} / \mathrm{A}$ are shown in Figure 1(b). For quality control, genotyping was repeated on a random $10 \%$ of the samples.

2.4. Statistical Analysis. A goodness-of-fit Chi-squared test was used to test for Hardy-Weinberg equilibrium (HWE) among the cancer-free controls. Study subjects who had smoked more than 100 cigarettes during their lifetime were 

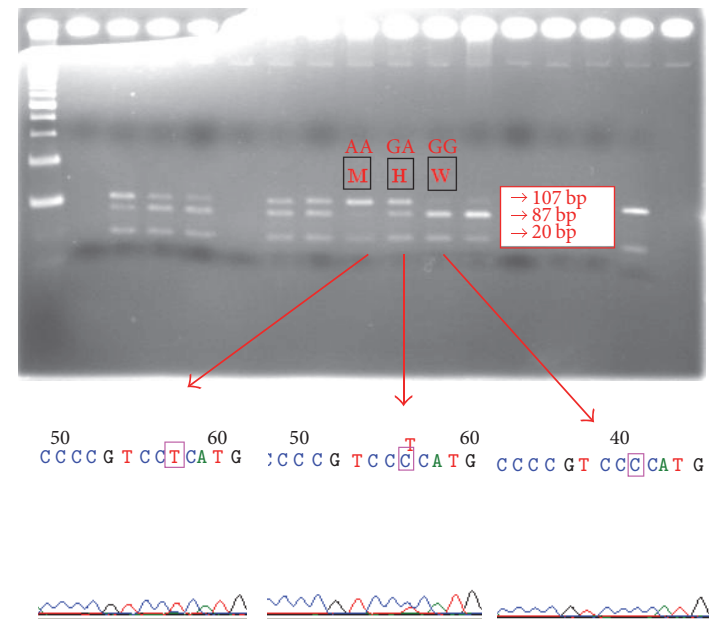

$\stackrel{2}{=}$

(a)
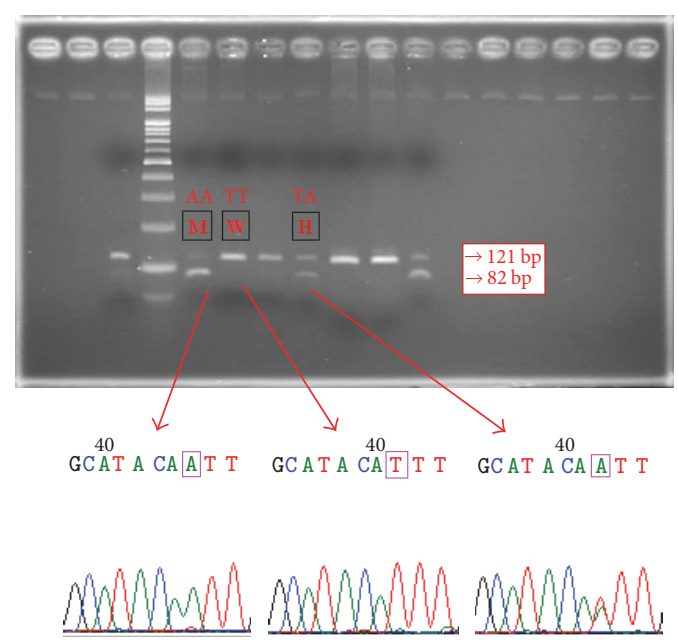

(b)

FiguRE 1: PCR-RFLP genotyping and sequencing of TNF- $\alpha-308$ G/A and $I L-8-251$ T/A. (a) Genotyping of TNF- $\alpha$-308 G/A. Arrows indicated location of AA, GA, and GG. (b) Genotyping of $I L-8-251$ T/A. Arrows indicated location of AA, TT, and TA.

regarded as people who had ever smoked, while those who had smoked fewer than 100 cigarettes were defined as people who had never smoked. The pack-years of cigarette smoking were calculated using the formula: pack-years $=($ cigarettes per day $\div 20) \times$ (number of years smoked). Because of dependent variable (UC) is binary, logistic regression is used to assess the association between UC and related risk factors. The combined effects of cumulative cigarette exposure, the TNF- $\alpha-308 \mathrm{~A} / \mathrm{A}$ genotype, and the $I L-8-251 \mathrm{~T} / \mathrm{T}$ genotype on the UC risk were estimated by odds ratios (ORs) and associated $95 \%$ confidence intervals (CIs) using a multivariateadjusted logistic regression. All data were analyzed using the Statistical Analysis Software for Windows, ver. 9.2 (SAS Institute, Cary, NC). $p$ values of $<0.05$ were considered statistically significant.

\section{Results}

No significant differences were found in age or gender between the UC cases and cancer-free controls. Occasional alcohol drinkers had a significantly lower OR for UC than people who had never consumed alcohol. After adjusting for age, gender, educational level, alcohol consumption, and other risk factors, people who had ever smoked had a significantly higher UC OR of 1.65 (95\% CI: 1.19 2.27) compared to people who had never smoked. Individuals who were exposed to environmental tobacco smoke had a significantly higher UC OR (1.68, 95\% CI: 1.22 2.30) than those with no exposure. Frequencies of the TNF- $\alpha-308$ A/A genotype and the $I L-8-251 \mathrm{~T} / \mathrm{T}$ genotype among controls fit HWE $\left(\chi^{2}=\right.$ $0.99, p>0.05 ; \chi^{2}=1.27, p>0.05$, resp.). The genotype distribution of the control did not show significant difference from the Hardy-Weinberg equilibrium values. Subjects who carried the TNF- $\alpha-308$ A/A genotype had a significantly increased OR for UC (3.56, 95\% CI: 1.03 12.28) compared to those with the G/G or G/A genotype. Subjects who carried the A/A genotype of the $I L-8-251 \mathrm{~T} / \mathrm{A}$ polymorphism had a significantly lower OR for UC $(0.52,95 \%$ CI: $0.27 \sim 0.99)$ than those carrying the $\mathrm{T} / \mathrm{T}$ genotype (data not shown).

After stratification by the TNF- $\alpha-308$ G/A and IL- 8 $251 \mathrm{~T} / \mathrm{A}$ genotypes, individual characteristics, including age, gender, educational level, cigarette smoking, environmental tobacco smoke exposure, and alcohol consumption, displayed no significantly different distributions in either of the polymorphism strata (Table 1). In terms of the effect of the cigarette smoking profile on the OR for UC, we found significantly increased ORs (95\% CI) for UC of 2.33 (1.52 3.55), 2.74 (1.77 4.24), and 2.64 (1.75 3.99) for subjects who had smoked more than 1 pack per day, individuals who had smoked for more than 31 years, and those who had smoked more than 18 pack-years, respectively (Table 2 ).

The joint effects of cigarette smoking, the TNF- $\alpha-308$ A/A genotype, and the $I L-8-251 \mathrm{~T} / \mathrm{T}$ genotype on the OR for UC after multivariate adjustment are shown in Figure 2. People who had ever smoked and who carried the A/A genotype of the TNF- $\alpha-308$ G/A polymorphism had a significantly higher UC OR of 10.25 than people who had never smoked and who carried the G/G or G/A genotype. In addition, people who had ever smoked who carried the T/T genotype of the $I L-8-251$ T/A polymorphism had a significantly increased UC OR of 3.08 compared to those who carried the T/A or A/A genotype.

Furthermore, we included three major risk factors (cumulative cigarette smoking, the TNF- $\alpha-308$ A/A genotype, and the $I L-8-251 \mathrm{~T} / \mathrm{T}$ genotype) in a combined analysis (Table 3). Compared to individuals who did had none of these risk factors, significantly increased ORs (95\% CI) for UC were 1.55 (1.03 2.35), 2.89 (1.70 4.93), and 3.77 (2.16 6.56) for study subjects with any one, any two, and all risk factors, respectively; these risk factors showed a significant dose-response relationship ( $p$ for trend $<0.0001$ ). 


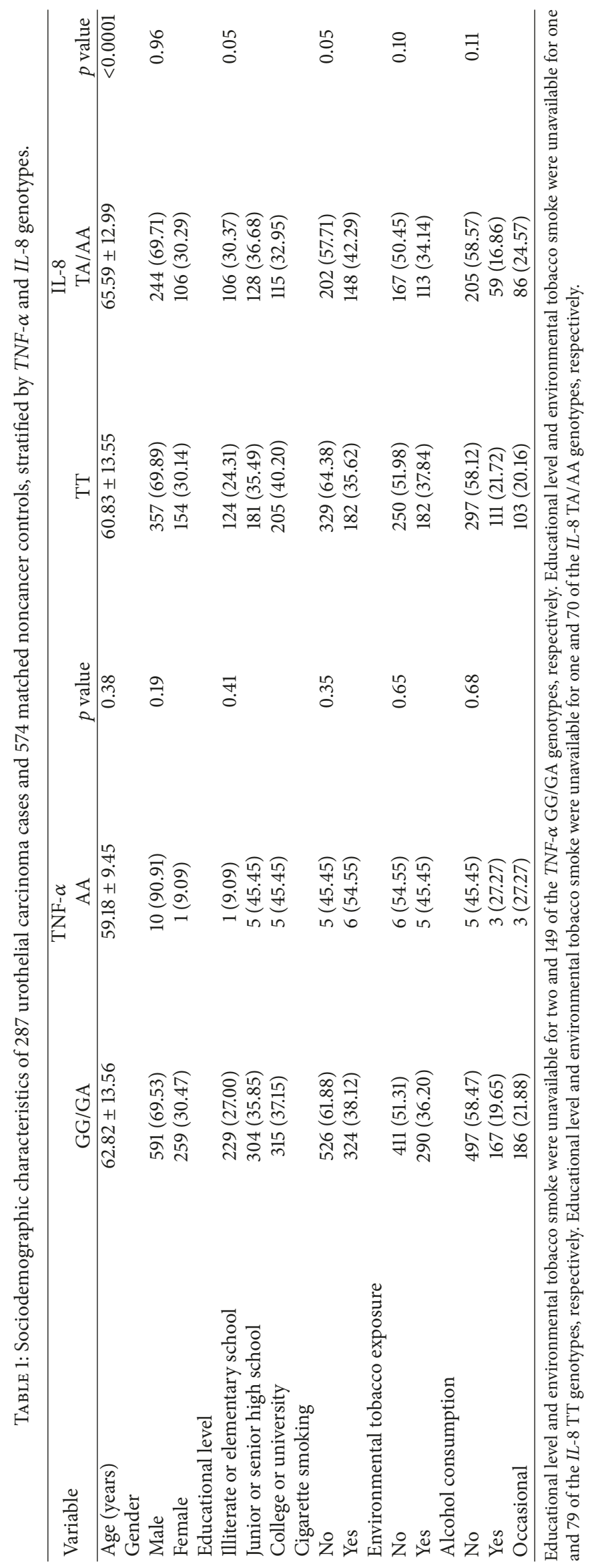


TABLE 2: Dose-response relationship between cigarette smoking profiles and the odds ratio (OR) for urothelial carcinoma (UC).

\begin{tabular}{|c|c|c|c|c|}
\hline & UC cases & Controls & $\begin{array}{c}\text { Age/sex-adjusted OR } \\
(95 \% \mathrm{CI})\end{array}$ & $\begin{array}{c}\text { Multivariate-adjusted } \\
\text { OR }(95 \% \text { CI }) \\
\end{array}$ \\
\hline \multicolumn{5}{|c|}{ Cigarette smoking (packs/day) } \\
\hline 0 & 152 & 379 & 1.00 & 1.00 \\
\hline $0 \sim 1$ & 108 & 169 & $1.69(1.10 \sim 2.61)^{*}$ & $1.90(1.19 \sim 3.03)^{*}$ \\
\hline$>1$ & 27 & 26 & $2.39(1.62 \sim 3.54)^{*}$ & $2.33(1.52 \sim 3.55)^{*}$ \\
\hline \multicolumn{5}{|c|}{ Cigarette smoking (years) } \\
\hline 0 & 153 & 379 & 1.00 & 1.00 \\
\hline $0 \sim 31$ & 49 & 101 & $1.46(0.93 \sim 2.27)$ & $1.49(0.92 \sim 2.39)$ \\
\hline$>31$ & 85 & 94 & $2.59(1.74 \sim 3.86)^{*}$ & $2.74(1.77 \sim 4.24)^{*}$ \\
\hline \multicolumn{5}{|c|}{ Cigarette smoking (pack years) } \\
\hline 0 & 153 & 379 & 1.00 & 1.00 \\
\hline $0 \sim 18$ & 35 & 81 & $1.19(0.73 \sim 1.94)$ & $1.30(0.78 \sim 2.19)$ \\
\hline$>18$ & 99 & 114 & $2.62(1.80 \sim 3.81)^{*}$ & $2.64(1.75 \sim 3.99)^{*}$ \\
\hline
\end{tabular}

Multivariate ORs were adjusted for age, gender, educational level, and alcohol consumption. ${ }^{*} p<0.05$. CI: confidence interval.

TABLE 3: Adjusted odds ratios (ORs) of urothelial carcinoma risk by cumulative cigarette exposure, the tumor necrosis factor (TNF)- $\alpha-308$ A/A genotype, and the interleukin (IL)-8 -251 T/T genotype.

\begin{tabular}{lcr}
\hline Risk factors & Age/sex-adjusted OR (95\% CI) & Multivariate-adjusted OR (95\% CI) \\
\hline None & 1.00 & 1.00 \\
Any one risk factor & $1.47(0.99 \sim 2.19)$ & $1.55(1.03 \sim 2.35)^{*}$ \\
Any two risk factors & $2.69(1.63 \sim 4.43)^{*}$ & $2.89(1.70 \sim 4.93)^{*}$ \\
All three risk factors & $3.61(2.15 \sim 6.07)^{*}$ & $3.77(2.16 \sim 6.56)^{*}$ \\
& $p_{\text {trend }}<0.0001$ & $p_{\text {trend }}<0.0001$ \\
\hline
\end{tabular}

The three risk factors were cumulative cigarette exposure,the TNF- $\alpha-308$ A/A genotype, and the IL-8 -251 T/T genotype. Multivariate ORs were adjusted for age, gender, educational level, and alcohol consumption. ${ }^{*} p<0.05$. CI: confidence interval.

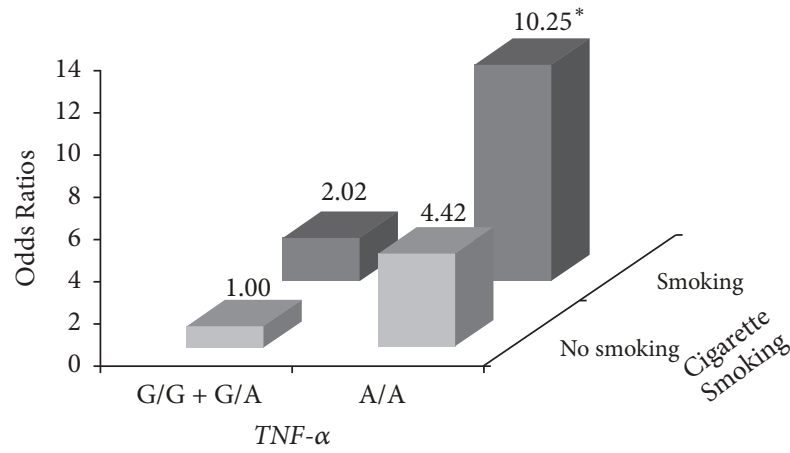

(a)

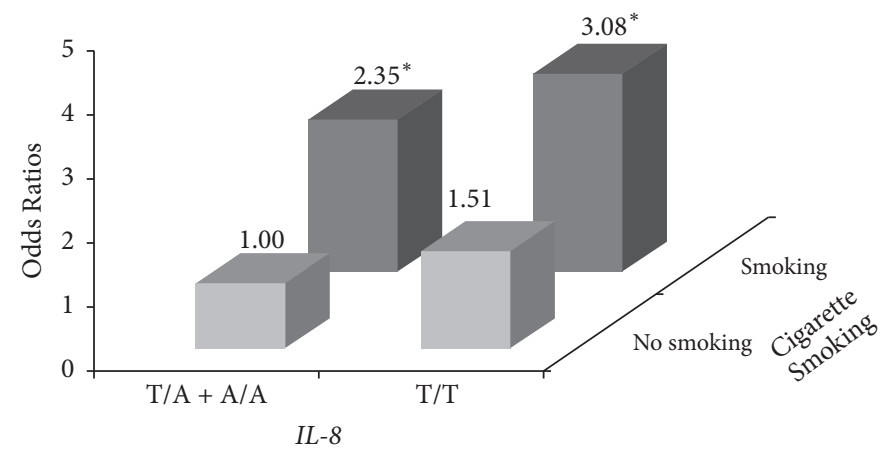

(b)

FIGURE 2: Joint effects of (a) the tumor necrosis factor $\alpha$ (TNF- $\alpha$ ) genotype and cigarette smoking and (b) the interleukin 8 (IL-8) genotype and cigarette smoking on the urothelial carcinoma (UC) risk after adjusting for age, gender, educational level, and alcohol consumption. ${ }^{*} p<0.05$.

\section{Discussion}

In this study, the risk of UC in smokers was 2 2.5-fold compared to nonsmokers. This result was consistent with a previous study [22]. In our previous study, there were no significant differences in the association of age and gender between UC cases and controls. UC conventional risk factors such as status smoking carried 1.65-fold risk [23]. Smokers or people who had ever smoked and carried TNF- $\alpha-308$ A/A genotype and the $I L-8-251 \mathrm{~T} / \mathrm{T}$ genotype had a higher risk of UC compared to nonsmokers. Gene-environment interactions are a potential way to identify individuals' risk. This study analyzed the combined effect of three major risk factors (high cumulative cigarette smoking, the TNF- $\alpha-308$ A/A genotype, and the $I L-8-251 \mathrm{~T} / \mathrm{T}$ genotype) and found dose-response relationships for UC risk in study subjects with 
any one risk factor, any two risk factors, and all three risk factors.

Functional polymorphisms of inflammatory genes can affect cytokine production. A previous study reported that the TNF- $\alpha-308 \mathrm{G} / \mathrm{A}$ polymorphism contributes to carcinogenesis and that the variant $A$ allele was associated with increased TNF- $\alpha$ expression [14]. A Korean study also reported that the TNF- $\alpha-308$ G/A polymorphism was significantly associated with the tumor stage and grade of bladder cancer [24]. In this study, subjects who carried the A/A genotype of the TNF- $\alpha$ -308 G/A polymorphism had a significantly higher OR for UC than those with the G/G or G/A genotype. These results suggest that the TNF- $\alpha-308$ G/A polymorphism might regulate angiogenesis, which plays a role in the invasion and metastasis of various tumors. However, another study did not report the same findings in urinary stone diseases or bladder cancer in Taiwan [25].

Compared to previous studies, differences in genotype frequencies may have been due to ethnic variations. The A/A genotype frequency of TNF- $\alpha$ in the case and control groups of this study was $2.23 \%$ and $0.67 \%$, respectively, and the TNF- $\alpha \mathrm{A} / \mathrm{A}$ genotype carried a 3.56-fold risk of UC. A meta-analysis study showed that the A/A genotype frequency of TNF- $\alpha$ in cervical cancer cases and controls was $0.97 \%$ $7.33 \%$ and $0 \% \sim 6.78 \%$, respectively. The TNF- $\alpha$ A/A genotype significantly elevating risks of cervical cancer was found in a Caucasian population (OR: 2.09, 95\% CI: 1.34 3.25) [26]. Although the TNF- $\alpha$ A/A genotype frequency was very low in Japanese (141 bladder cancer patients and 173 control subjects), the allelic frequency of patients (3.5\%) was higher than that in controls (0.6\%) [27]. A previous study indicated that cigarette smoking activates systemic inflammation and upregulates TNF- $\alpha$ expression in an animal model [8]. Another study also found that the circulating TNF- $\alpha$ level was higher in people who had ever smoked compared to people who had never smoked [17]. A study also showed that the A allele polymorphism of TNF- $\alpha-308$ genotypes was associated with HCC risk in Taiwan males who were exposed to cigarette and alcohol [28]. In the present study, we observed a significant combined effect of cigarette smoking and the A/A genotype of the TNF- $\alpha-308$ polymorphism on the OR (10.25) for UC. This finding suggests that the TNF- $\alpha$ 308 G/A polymorphism can modify the OR for UC, especially in people who had ever smoked.

IL-8 plays a critical role in inflammation. A common single-nucleotide polymorphism at the -251 position of the $I L-8$ promoter region can influence its expression and may increase one's susceptibility to bladder cancer [29]. In the present study, subjects who carried the A/A genotype had a significantly decreased OR for UC. Previous studies explored the association between the $I L-8-251 \mathrm{~T} / \mathrm{A}$ polymorphism and various cancer risks, but the findings were inconsistent $[29,30]$. Some researchers reported that the $I L-8-251 \mathrm{~A}$ allele has significantly higher promoter activity than the $-251 \mathrm{~T}$ allele [31]. However, another study reported that the $I L-8$ $251 \mathrm{~T}$ allele had significantly higher transcriptional activity than the $-251 \mathrm{~A}$ allele of the $I L-8$ gene [32]. In addition, previous studies indicated that cigarette smoking causes chronic inflammation characterized by increased levels of cytokines, such as IL-8 $[10,11]$. IL-8 plays a role in assisting cancer cells to eschew stress and induce apoptosis and is also involved in angiogenesis, tumor growth, and metastasis [33]. A meta-analysis showed that IL-8 -251A/T polymorphism has significantly elevated risks of cancer in Asian population [34]. It is possible that the increased IL-8 expression induced by cigarette smoking may modify the carcinogenesis of UC. In the present study, we found a significant joint effect of cigarette smoking and the T/T genotype of the $-251 \mathrm{~T} / \mathrm{A}$ polymorphism on the OR for $\mathrm{UC}(\mathrm{OR}=3.08)$. This suggests that the $-251 \mathrm{~T} / \mathrm{A}$ polymorphism can modify the UC risk, especially in people who have ever smoked.

Single-nucleotide polymorphisms of genes can increase disease susceptibilities via affect gene expression. Silico analysis is a novel computational approach to identify potential SNPs causing transcription factor binding affinities to change and influence regulatory functions of genes [35]. Since polymorphisms have been identified and their transition is considered to be an important enhancer of transcriptional activation associated with elevated levels genes expression, structural analysis study showed that TNF- $\alpha$ protein stability was impacted by amino acid residue substitutions of P84L (rs4645843) and A94T (rs1800620) [36]. The effect of IL-8 SNPs and protein structure in disease risk was also conducted by silico analysis [37]. Although the level of confidence is too high in some cases, it is still too low for clinical purpose, which is a main limitation of silico analysis programs [38].

Our study has some limitations. We only investigated one polymorphism located in the promoter region, which might not entirely account for the functions of the $T N F-\alpha$ and $I L$ 8 genes. More functional polymorphisms of the TNF- $\alpha$ and IL-8 genes should be included in future analyses with larger samples to validate our findings. Other proinflammatory cytokines such as NF- $\kappa \mathrm{B}$ also need to be further investigated. In addition, interactions of cigarette smoking and other cytokines on the UC risk should be explored in the future.

\section{Conclusions}

We found a dose-response relationship between the number of risk factors and the OR for UC. These findings suggest that individuals who carry high-risk genotypes, including the TNF- $\alpha-308$ A/A genotype and the $I L-8-251$ T/T genotype, and who experience cumulative cigarette smoking have a significantly increased OR for UC in a dose-response manner.

\section{Data Availability}

The data used to support the findings of this study are included within the article.

\section{Conflicts of Interest}

The authors have disclosed all financial and interpersonal relationships that present potential conflicts of interest.

\section{Authors' Contributions}

Dr. Chia-Chang Wu and Dr. Yung-Kai Huang contributed equally to this work. 


\section{Acknowledgments}

This study was supported by Grants (NSC90-2320-B038-021, NSC91-3112-B-038-0019, NSC92-3112-B-038-001, NSC93-3112-B-038-001， NSC94-2314-B-038-023， NSC952314-B-038-007, NSC96-2314-B038-003, NSC97-2314-B-038015-MY3, NSC100-2314-B-038 -026, and NSC101-2314-B038-051-MY3) from National Science Council, Taiwan, and by Grants (MOST103-2314-B-038-021-MY2 (1-2), MOST1032314-B-038-021-MY2 (2-2), and MOST 105-2314-B-038-082) from Ministry of Science and Technology, Taiwan, to YM-H.

\section{References}

[1] D. Volanis, T. Kadiyska, A. Galanis, D. Delakas, S. Logotheti, and V. Zoumpourlis, "Environmental factors and genetic susceptibility promote urinary bladder cancer," Toxicology Letters, vol. 193, no. 2, pp. 131-137, 2010.

[2] C. Samanic, M. Kogevinas, M. Dosemeci et al., "Smoking and bladder cancer in Spain: Effects of tobacco type, timing, environmental tobacco smoke, and gender," Cancer Epidemiology, Biomarkers \& Prevention, vol. 15, no. 7, pp. 1348-1354, 2006.

[3] S. S. Hecht, "Tobacco carcinogens, their biomarkers and tobacco-induced cancer," Nature Reviews Cancer, vol. 3, no. 10, pp. 733-744, 2003.

[4] S. S. Hecht, "Human urinary carcinogen metabolites: Biomarkers for investigating tobacco and cancer," Carcinogenesis, vol. 23, no. 6, pp. 907-922, 2002.

[5] IARC, Monographs on the Evaluation of the Carcinogenic Risk of Chemicals to Humans: Tobacco Smoking, WHO, Lyon, France, 1986.

[6] N. Wang, R. Zhou, C. Wang et al., “-251 T/A polymorphism of the interleukin-8 gene and cancer risk: a HuGE review and meta-analysis based on 42 case-control studies," Molecular Biology Reports, vol. 39, no. 3, pp. 2831-2841, 2012.

[7] P. Zhou, G.-Q. Lv, J.-Z. Wang et al., "The TNF-Alpha-238 polymorphism and cancer risk: a meta-analysis," PLOS ONE, vol. 6, no. 7, Article ID e22092, 2011.

[8] Y.-T. Li, B. He, and Y.-Z. Wang, "Exposure to cigarette smoke upregulates AP-1 activity and induces TNF-alpha overexpression in mouse lungs," Inhalation Toxicology, vol. 21, no. 7, pp. 641-647, 2009.

[9] K. Taniguchi, S. Koga, M. Nishikido et al., "Systemic immune response after intravesical instillation of bacille CalmetteGuerin (BCG) for superficial bladder cancer," Clinical \& Experimental Immunology, vol. 115, no. 1, pp. 131-135, 1999.

[10] S. E. Tanni, N. R. Pelegrino, A. Y. Angeleli, C. Correa, and I. Godoy, "Smoking status and tumor necrosis factor-alpha mediated systemic inflammation in COPD patients," Journal of Inflammation, vol. 7, article 29, 2010.

[11] H.-Y. Wang, Y.-N. Ye, M. Zhu, and C.-H. Cho, "Increased interleukin-8 expression by cigarette smoke extract in endothelial cells," Environmental Toxicology and Pharmacology, vol. 9, no. 1-2, pp. 19-23, 2000.

[12] B. B. Aggarwal, S. Shishodia, S. K. Sandur, M. K. Pandey, and G. Sethi, "Inflammation and cancer: how hot is the link?" Biochemical Pharmacology, vol. 72, no. 11, pp. 1605-1621, 2006.

[13] H. P. Marsh, N. A. Haldar, M. Bunce et al., "Polymorphisms in tumour necrosis factor (TNF) are associated with risk of bladder cancer and grade of tumour at presentation," British Journal of Cancer, vol. 89, no. 6, pp. 1096-1101, 2003.
[14] D. Ahirwar, P. Kesarwani, P. K. Manchanda, A. Mandhani, and R. D. Mittal, "Anti- and proinflammatory cytokine gene polymorphism and genetic predisposition: association with smoking, tumor stage and grade, and bacillus Calmette-Guérin immunotherapy in bladder cancer," Cancer Genetics and Cytogenetics, vol. 184, no. 1, pp. 1-8, 2008.

[15] K. M. Kroeger, J. H. Steer, D. A. Joyce, and L. J. Abraham, "Effects of stimulus and cell type on the expression of the -308 tumour necrosis factor promoter polymorphism," Cytokine, vol. 12, no. 2, pp. 110-119, 2000.

[16] C. Shen, H. Sun, D. Sun et al., "Polymorphisms of tumor necrosis factor-alpha and breast cancer risk: A meta-analysis," Breast Cancer Research and Treatment, vol. 126, no. 3, pp. 763770, 2011.

[17] J. J. Yang, K.-P. Ko, L. Y. Cho et al., "The role of TNF genetic variants and the interaction with cigarette smoking for gastric cancer risk: a nested case-control study," BMC Cancer, vol. 9, article 238, 2009.

[18] A. Loktionov, "Common gene polymorphisms, cancer progression and prognosis," Cancer Letters, vol. 208, no. 1, pp. 1-33, 2004.

[19] S. K. Ahuja, T. Özçelik, A. Milatovitch, U. Francke, and P. M. Murphy, "Molecular evolution of the human interleukin-8 receptor gene cluster," Nature Genetics, vol. 2, no. 1, pp. 31-36, 1992.

[20] I. Duarte, A. Santos, H. Sousa et al., "G-308A TNF- $\alpha$ polymorphism is associated with an increased risk of invasive cervical cancer," Biochemical and Biophysical Research Communications, vol. 334, no. 2, pp. 588-592, 2005.

[21] E. Vairaktaris, C. Yapijakis, Z. Serefoglou et al., "The interleukin-8 $(-251 \mathrm{~A} / \mathrm{T})$ polymorphism is associated with increased risk for oral squamous cell carcinoma," European Journal of Surgical Oncology, vol. 33, no. 4, pp. 504-507, 2007.

[22] P. Vineis, G. Talaska, C. Malaveille et al., "DNA adducts in urothelial cells: Relationship with biomarkers of exposure to arylamines and polycyclic aromatic hydrocarbons from tobacco smoke," International Journal of Cancer, vol. 65, no. 3, pp. 314316,1996

[23] C.-C. Wu, Y.-K. Huang, C.-J. Chung et al., "Polymorphism of inflammatory genes and arsenic methylation capacity are associated with urothelial carcinoma," Toxicology and Applied Pharmacology, vol. 272, no. 1, pp. 30-36, 2013.

[24] P. Jeong, E.-J. Kim, E.-G. Kim, S.-S. Byun, C. S. Kim, and W.J. Kim, "Association of bladder tumors and GA genotype of 308 nucleotide in tumor necrosis factor-alpha promoter with greater tumor necrosis factor-alpha expression," Urology, vol. 64, no. 5, pp. 1052-1056, 2004.

[25] F.-J. Tsai, H.-F. Lu, L.-S. Yeh, C.-D. Hsu, and W.-C. Chen, "Lack of evidence for the association of tumor necrosis factor-alpha gene promoter polymorphism with calcium oxalate stone and bladder cancer patients," Urolithiasis, vol. 29, no. 6, pp. 412-416, 2001.

[26] F. Pan, J. Tian, C. Ji et al., "Association of TNF- $\alpha-308$ and -238 Polymorphisms with Risk of Cervical Cancer: A Meta-analysis," Asian Pacific Journal of Cancer Prevention, vol. 13, no. 11, pp. 5777-5783, 2012.

[27] N. Nonomura, T. Tokizane, M. Nakayama et al., "Possible correlation between polymorphism in the tumor necrosis factor-beta gene and the clinicopathological features of bladder cancer in Japanese patients," International Journal of Urology, vol. 13, no. 7, pp. 971-976, 2006. 
[28] M. D. Yang, C. M. Hsu, W. S. Chang et al., "Tumor Necrosis Factor-alpha Genotypes Are Associated with Hepatocellular Carcinoma Risk in Taiwanese Males, Smokers and Alcohol Drinkers," Anticancer Res, vol. 35, no. 10, pp. 5417-5423, 2015.

[29] D. K. Ahirwar, A. Mandhani, and R. D. Mittal, "IL-8 -251 T > A Polymorphism is associated with bladder cancer susceptibility and outcome after BCG immunotherapy in a northern Indian cohort," Archives of Medical Research, vol. 41, no. 2, pp. 97-103, 2010.

[30] L.-B. Gao, X.-M. Pan, J. Jia et al., "IL-8 -251A/T polymorphism is associated with decreased cancer risk among population-based studies: Evidence from a meta-analysis," European Journal of Cancer, vol. 46, no. 8, pp. 1333-1343, 2010.

[31] M. Ohyauchi, A. Imatani, M. Yonechi et al., "The polymorphism interleukin $8-251 \mathrm{~A} / \mathrm{T}$ influences the susceptibility of Helicobacter pylori related gastric diseases in the Japanese population," Gut, vol. 54, no. 3, pp. 330-335, 2005.

[32] W.-P. Lee, D.-I. Tai, K.-H. Lan et al., "The -251T allele of the interleukin- 8 promoter is associated with increased risk of gastric carcinoma featuring diffuse-type histopathology in Chinese population," Clinical Cancer Research, vol. 11, no. 18, pp. 6431-6441, 2005.

[33] D. J. Waugh and C. Wilson, "The interleukin-8 pathway in cancer," Clinical Cancer Research, vol. 14, no. 21, pp. 6735-6741, 2008.

[34] Z. Wang, Y. Liu, L. Yang, S. Yin, R. Zang, and G. Yang, "The polymorphism interleukin-8 -251A/T is associated with a significantly increased risk of cancers from a meta-analysis," Tumor Biology, vol. 35, no. 7, pp. 7115-7123, 2014.

[35] R. Li, D. Zhong, R. Liu et al., "A novel method for in silico identification of regulatory SNPs in human genome," Journal of Theoretical Biology, vol. 415, pp. 84-89, 2017.

[36] B. Dabhi and K. N. Mistry, "In silico analysis of single nucleotide polymorphism (SNP) in human TNF- $\alpha$ gene," Meta Gene, vol. 2, pp. 586-595, 2014.

[37] T. C. Dakal, D. Kala, G. Dhiman, V. Yadav, A. Krokhotin, and N. V. Dokholyan, "Predicting the functional consequences of nonsynonymous single nucleotide polymorphisms in IL8 gene," Scientific Reports, vol. 7, no. 1, article no. 6525, 2017.

[38] D. Tchernitchko, M. Goossens, and H. Wajcman, "In silico prediction of the deleterious effect of a mutation: Proceed with caution in clinical genetics," Clinical Chemistry, vol. 50, no. 11, pp. 1974-1978, 2004. 


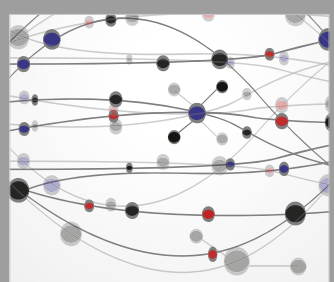

The Scientific World Journal
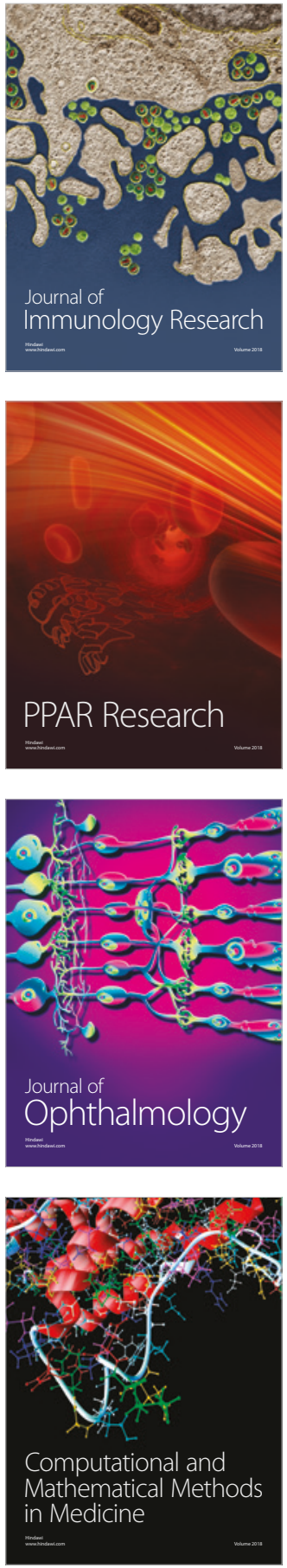

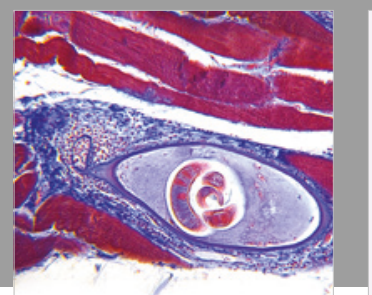

Gastroenterology Research and Practice

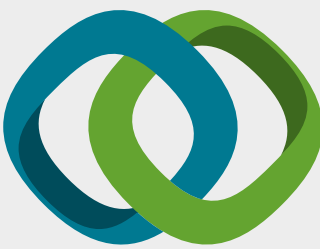

\section{Hindawi}

Submit your manuscripts at

www.hindawi.com
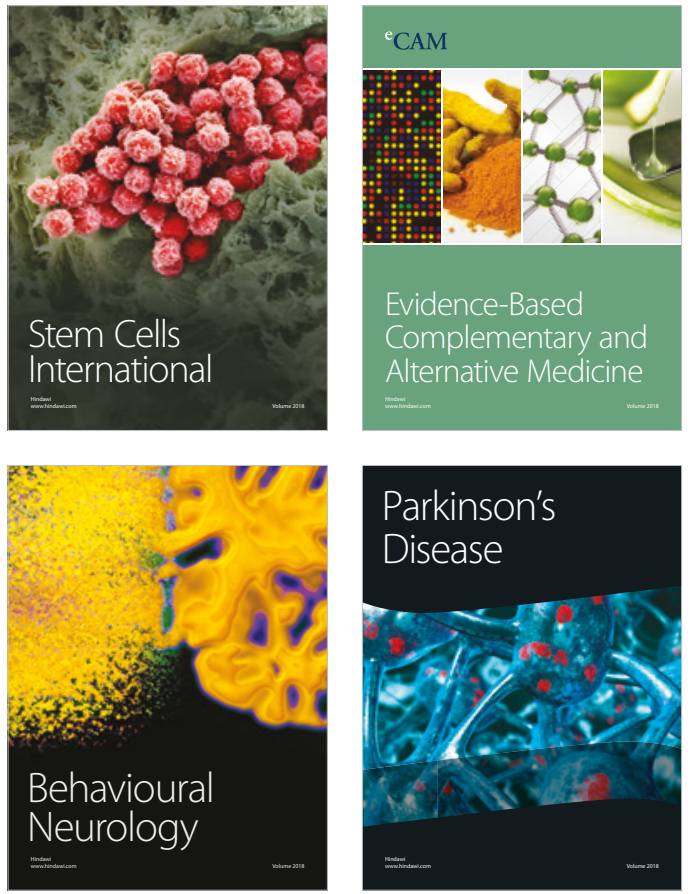

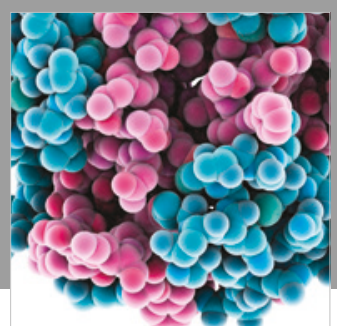

ournal of

Diabetes Research

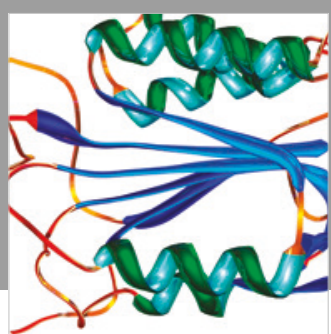

Disease Markers
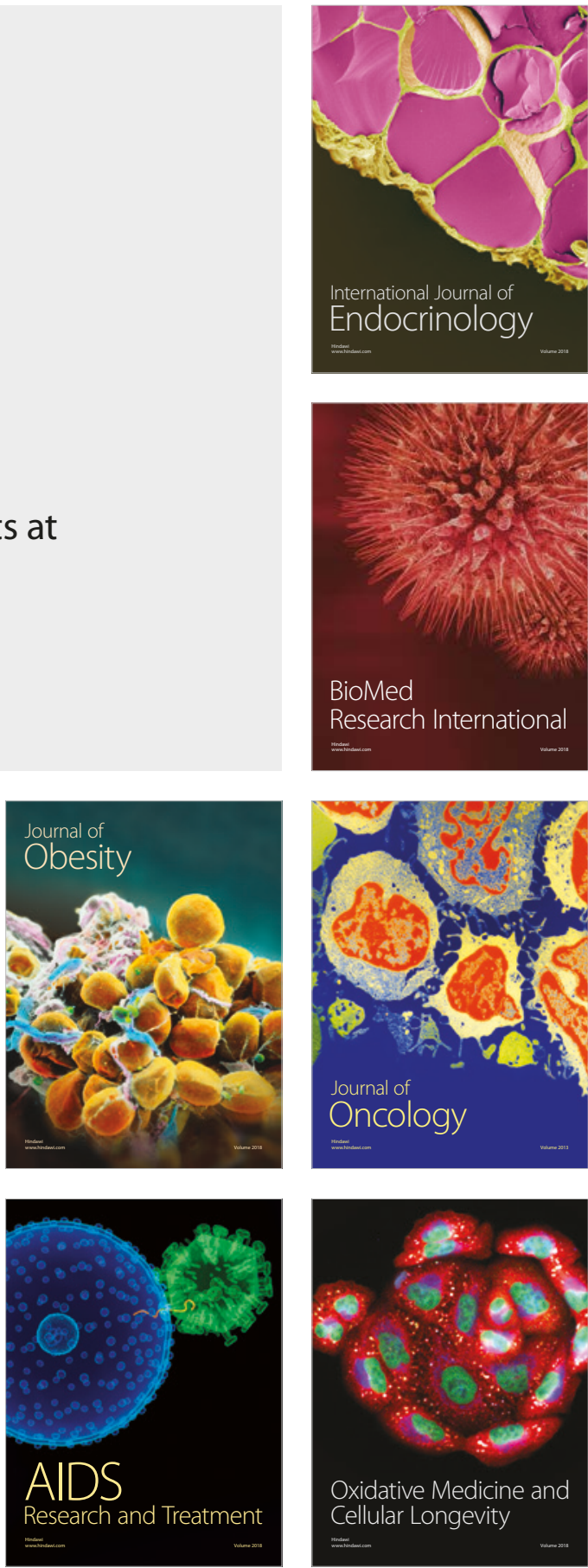